\title{
Negative Pressure Wound Therapy for Necrotizing Fasciitis and Compartment Syndrome of the Upper Extremity - a case report
}

\author{
Attila Enyedi, Gábor Mudriczki, Tamás Bazsó, Ferenc Győry, Zsolt Susán, László Damjanovich, Zsolt \\ Szentkereszty
}

\section{CASE REPORT}

\begin{abstract}
Background: Necrotizing fasciitis (NF) is a lifethreatening infection of the subcutaneous tissues that spreads along the underlying fascia. Despite the early and aggressive surgical fasciotomy and necrectomy, its mortality rate is still high. In NF the negative pressure wound therapy (NPWT) shows good effects on wound healing and on the primary closure of the concomitant extended tissue defects.

Case report: A 32-year-old male patient was admitted with a four-day history of fever $\left(39.1^{\circ} \mathrm{C}\right)$, pain, swelling, erythema of the right elbow and the upper arm. On admission, extensive erythema and swelling were seen on the right forearm, arm, and the pectoral region with superficial skin bullae. Based on the clinical symptoms and laboratory tests immediate surgery was indicated. Extended fasciotomy and necrosectomy were performed on the full extremity and pectoral region. Negative pressure wound therapy was started immediately afterward with $-120 \mathrm{mmHg}$ concomitantly with antibiotic therapy.

Results: After five cycles of NPWT the patient recovered without needing any plastic surgical intervention. The functional and aesthetic results were excellent.

Conclusion: In the case of extended NF of the upper extremity the aggressive surgery and NPWT are relatively safe and effective.
\end{abstract}

Keywords-negative pressure wound therapy with installation, necrotizing fasciitis

\section{INTRODUCTION}

$\mathbf{N}$ ECROTIZING fasciitis (NF) is a relatively rare, but lifethreatening disease. The extremities are most commonly affected, followed by the trunk $!^{1 / 5}$ It has monobacterial ( $\beta$-hemolytic Streptococcus), polymicrobial or fungal etiology 1 4] 6 Local symptoms are pain, erythema, tenderness, swelling, induration, and crepitation! $\sqrt{36}$ In advanced cases, skin bullae and necrosis can develop. General symptoms are fever, hypotension, diaphoresis, and anxiety. ${ }^{[}$

The effective treatment based on the early diagnosis is the aggressive surgical debridement and fasciotomy, broadspectrum antibiotic therapy and supportive intensive care.

Manuscript received 30.12.2018; revised 04.04.2019. This work did not receive any financial support. Authors declare no conflict of interest.

Author affiliations: University of Debrecen, Faculty of Medicine, Inst. of Surgery 4032 Debrecen, Móricz Zs. krt 22, Hungary, (AE, GM, FG, ZS, LD, ZS)

; University of Debrecen, Faculty of Medicine, Dept. of Orthopaedic Surgery 4032 Debrecen, Nagyerdei krt. 98, Hungary, (TB)

*Correspondence to: Zsolt Szentkereszty: szentkerzs@gmail.com
Despite the adequate surgical and conservative therapy, NF has a high rate of mortality which ranges between $6-67 \%$ of cases as reported in the literature 2 [ 3: 5 , 60

The aggressive surgical debridement and fasciotomy often result in widespread tissue defects that need plastic surgical reconstruction! [1] 6 Negative pressure wound therapy is a frequently used method in the treatment of necrotizing fasciitis based on its advances in complex wound care. It has positive effects on wound healing, bacterial decontamination, removal of excessive, toxic fluid from the wound, angiogenesis and tissue perfusion! 1 10 In the last decade, the use of NPWT has become the gold standard in the treatment of NF1 3 3 4 6 6

We present a successful negative pressure wound therapy in case of extended necrotizing fasciitis and compartment syndrome of the right upper extremity.

\section{CASE REPORT}

Four-day history of pain, swelling, erythema of the right elbow and upper arm and fever $\left(39.1^{\circ} \mathrm{C}\right)$ were reported by the patient. On admission, extensive erythema and swelling were seen on the right forearm and arm. Some superficial skin bullae were also observed without skin necrosis (Fig. 1A-D). The extremity movement was painful and limited at the elbow, shoulder, and wrist. Laboratory results showed elevated white blood cell count $(16.08 \mathrm{G} / \mathrm{L})$, C-reactive protein $(513.67 \mathrm{mg} / \mathrm{L})$, procalcitonin $(6.4 \mu \mathrm{g} / \mathrm{L})$, creatinine $(147 \mu \mathrm{mol} / \mathrm{L})$, glucose $(10.6 \mathrm{mmol} / \mathrm{L})$ and decreased level of sodium $(132 \mathrm{mmol} / \mathrm{L})$ and hemoglobin $(135 \mathrm{~g} / \mathrm{L})$ were observed. The LRINEC (Laboratory Risk Indicator for Necrotizing Fasciitis) score was calculated to be 11 indicating a high risk of necrotizing fasciitis. The ultrasound and X-ray excluded osteomyelitis and deep vein or arterial obstruction. Chest CT scan showed only some axillary lymph node enlargement and no intrathoracic involvement. 32-year-old male patient reported shunt operations for hydrocephalus and inguinal hernia reconstruction in the childhood.

Broad-spectrum antibiotic therapy (imipenem with vancomycin) was started on admission and immediate surgery was indicated. The incision was started from the pectoral region to the carpal tunnel on the medial surface of the arm and forearm. The carpal tunnel was also explored (Fig. 


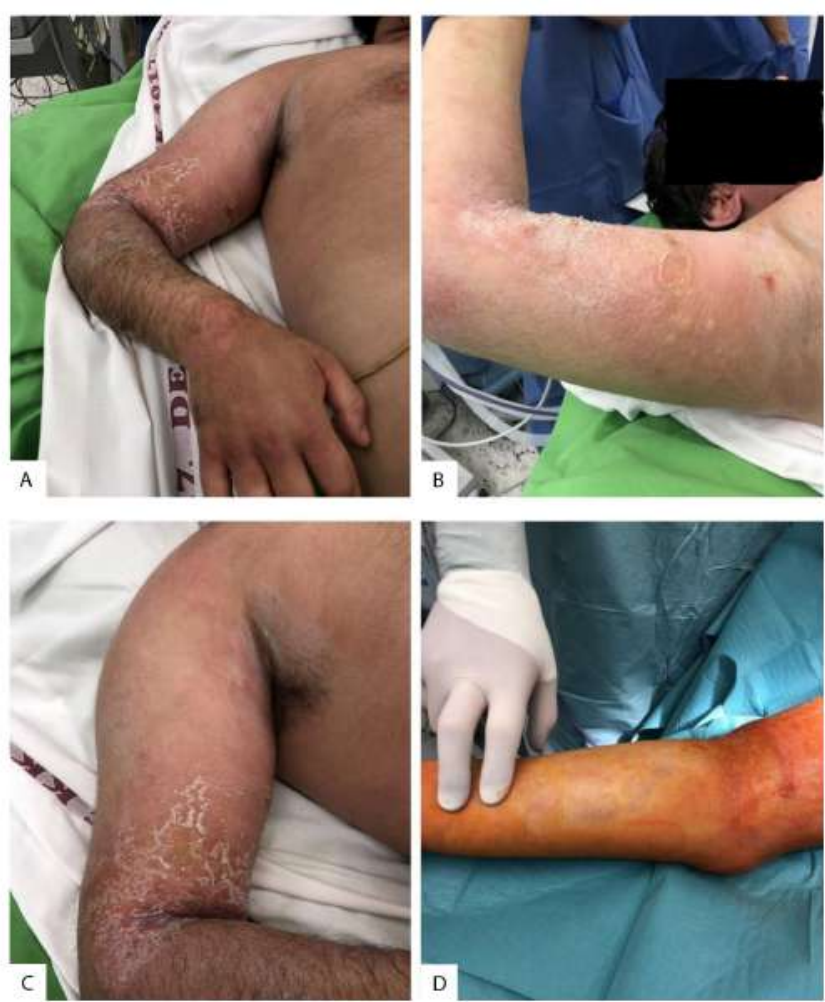

Figure 1. The view of the right arm and chest wall at admission
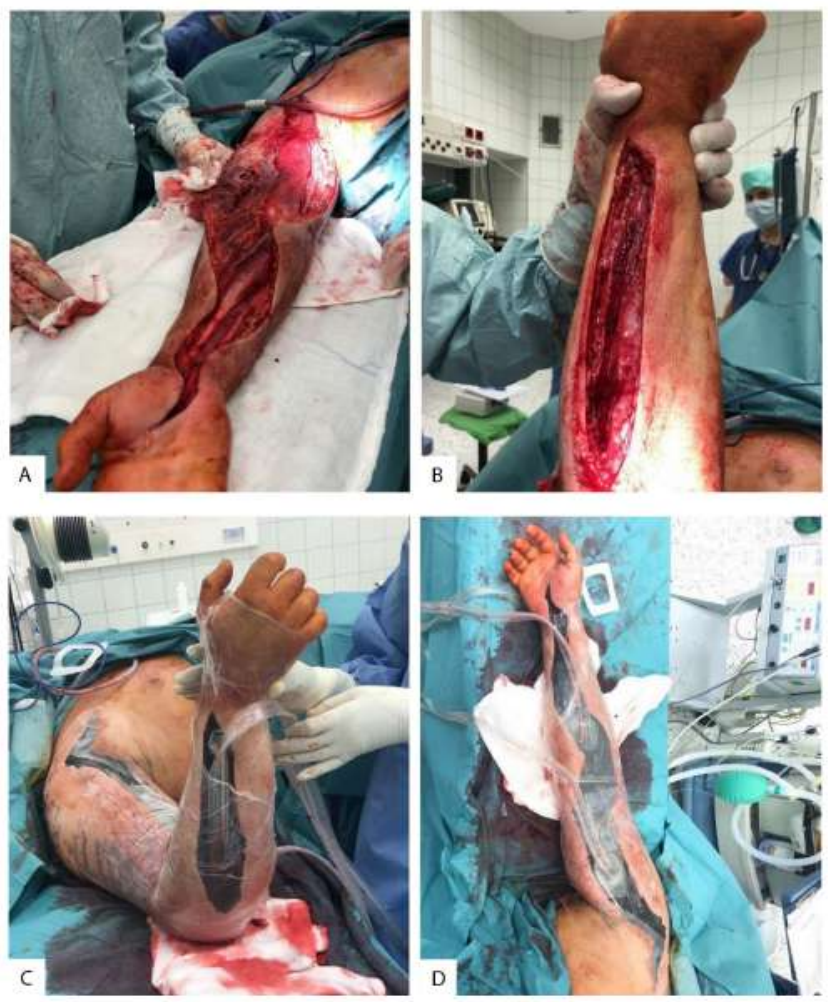

Figure 2. A: The volar incision, necrosectomy, B: The ulnar incision, C-D: The application of the NPWT
2A). Another ulnar incision was performed on the forearm. During the wide incisions, the finger test was positive (lack of resistance to blunt dissection). Blunt finger separation along the fascia plane was performed. The involved spaces were explored to decrease the pressure. The necrotic fascia was removed, and widespread debridement was performed (Fig. 2B). The margin of fascia resection was the visual assessment of tissue viability and satisfactory bleeding from the edges of resection. During the first dressing change, necrosectomy was necessary.

The surgical site was washed out with high-pressure saline solution. Macroscopically total debridement was possible so negative pressure wound therapy (Vivano $\mathrm{Med}^{\circledR}$, Paul Hartmann AG. Germany) was inserted into the surgical site. Shallow, superficial $(16 \mathrm{~mm}$ ) foam was used partly manually adapted (Fig. 2C-D). Continuous suction with $-120 \mathrm{mmHg}$ pressure was started and the patient's local and general status was closely observed. Intensive care was performed, the hypotension was treated with Arterenol. On the second postoperative day the patient was extubated, the circulatory support was finished. The laboratory tests began to normalize. Locally there were no signs for the spread of the disease so the first dressing was only performed on the 4th postoperative day. Only the first two dressing changes were made in general anesthesia.

The first 3 days for analgesia Würzburg cocktail (400 mg tramadol, $4 \mathrm{~g}$ metamizole sodium, and $2.5 \mathrm{mg}$ droperidol) in injection pump for 24 hours was administered. After that nalbuphine $10 \mathrm{mg} / \mathrm{ml}$ was required and at discharge diclofenac $100 \mathrm{mg} /$ day twice daily was administered.

After the 5th day, the patient was transferred from ICU to the general department. The wound surface was clean, there was no new necrosis and tissue granulation was observed. 4 more dressing changes were performed on every fourth day. On the first postoperative day, the amount of exudation was approximately $800 \mathrm{ml}$. On the $2 \mathrm{nd}$ postoperative day, it was $400 \mathrm{ml}$ and $150 \mathrm{ml}$ on the 3rd postoperative day. In addition, 5 NPWT cycles were conducted and 2 negative pressure devices were used in consecutive dressing changes. After the second NPWT, the wound started to close progressively with single sutures (Fig. 3A-D). There was no necessity for skin preparation during closure of skin wound. The short forearm's wound was closed first followed by the large one. The result of bacterial culture was Streptococcus pyogenes, without anaerobic bacterial and fungal infection. Empirically clindamycin (600 mg three times daily) and penicillin-G (6 times $4 \mathrm{MU}$ ) therapy were used for 9 days. Based on microbiological culture results the antibiotic therapy was changed to intravenous amoxicillin with clavulanic acid 3 times $1.2 \mathrm{~g}$ for 10 days followed by oral therapy for 7 days.

The dysfunction of the 4th finger became obvious during the treatment. Systematic (full-body) physiotherapy was performed from the 3rd postoperative day. The involved extremity was not spared from it, additional passive rehabilitation and manual lymphatic massage were performed at the beginning of each therapy session and the patient was transferred from ICU to the general department. The patient did not report any adverse reactions other than the 

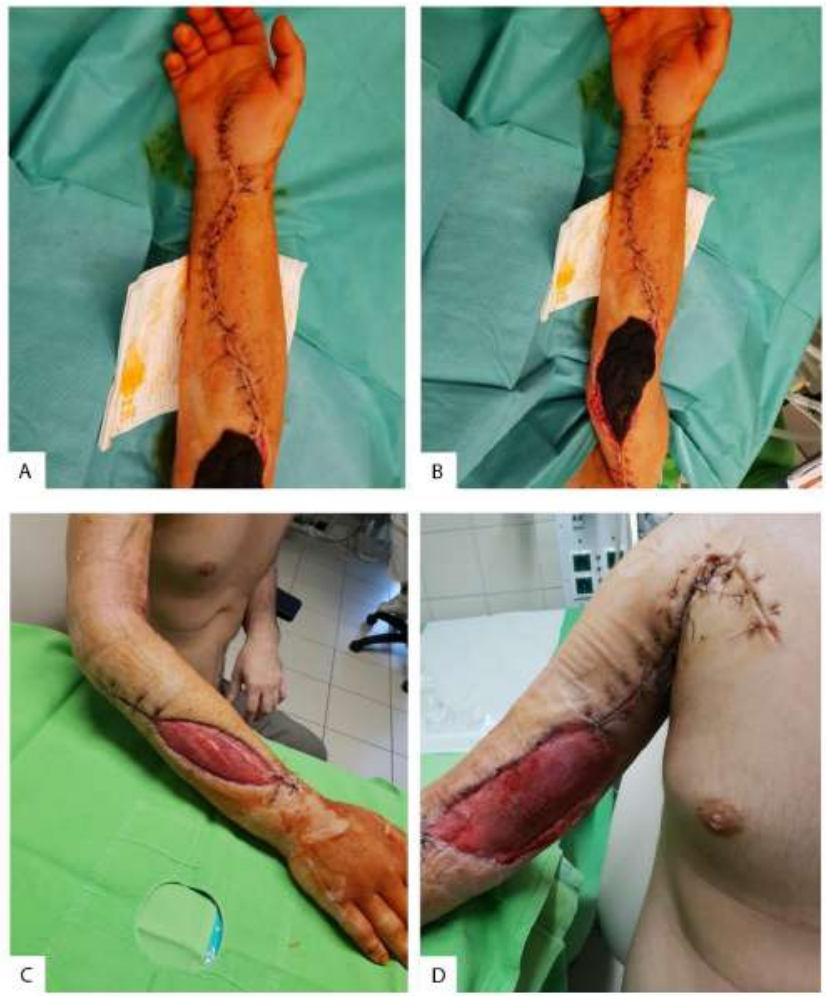

Figure 3. A-D: During the dressing changes the wounds were closed step by step from the edges to the center.

dysfunction of his 4 th finger.

On the 24th postoperative day, the patient was discharged. The residual $3 \mathrm{~cm}$ by $6 \mathrm{~cm}$ skin defect which was treated conservatively (Fig. 4A-D). The sensory and functional status of the extremity was nearly normal. After 30 day followup the patient was free of complaints, the function of the right upper extremity was normal with the except of his 4th finger's palmar flexion which was restricted to $30 \%$.

\section{DISCUSSION}

Necrotizing fasciitis is a life-threatening severe infection characterized by extensive soft tissue necrosis along fascial planes involving the subcutaneous region and skin.115 11 NF can appear on various body regions, but most often on the extremities. 34 11 It is often caused by monobacterial (Streptococcus pyogenes) infection but polymicrobial or fungal origins are not rare either $2 \sqrt{4} 6 \sqrt[8]{8}$ Thrombocytopenia, advanced age, liver diseases, acute renal failure, low serum albumin level, and smoking are risk factors for $\mathrm{NF}$ and increase mortality rate 4.6

The clinical complaints are the pain, swelling, erythema, tenderness and due to the generalization of the local infection fever and signs of sepsis. In advanced cases, skin bullae and necrosis can develop? 40 In advanced cases, anxiety can develop as a sign of generalized septic-toxic signs. $\frac{8}{6}$ When the disease is localized in the extremities, concomitant compartment syndrome is often observed ${ }^{4}$ The early diagnosis
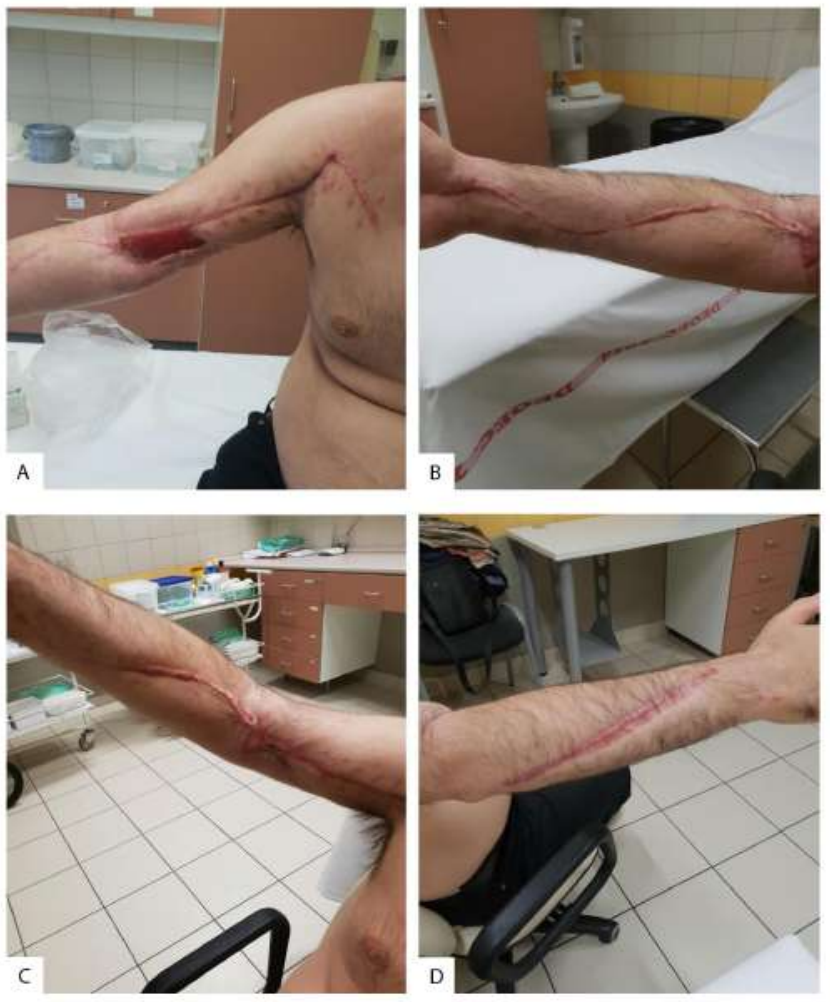

Figure 4. A: The skin defect on discharge, B-D: The final view of the upper extremity after a 30-day followup

is a crucial step in the treatment algorithm. Wong et al. proposed a scoring system (LRINEC) for early diagnosis of the disease ${ }^{5}$ based on the level of C-reactive protein, white blood cell count, hemoglobin, sodium, creatinine, and glucose 5 In the presented case the LRINEC score was 11. The intraoperative diagnosis was confirmed by grey colorization and necrotic deep fascia (without bleeding), lack of resistance during blunt dissection and presence of pus with odor 1. 2.5

The successful treatment is often based on the early aggressive surgical debridement, fasciotomy, and supportive therapy! 10 [ 90.11 Broad-spectrum antibiotics and intensive care assistive measures are needed for stabilizing the vital functions of the severely septic patient 1, 6, 11 During the conventional surgical treatment, wound dressings should be changed at least daily. Due to the aggressive debridement, extensive tissue defect may remain as an adverse therapy outcome that may in the future need a reconstructive plastic surgical intervention. 6 6. 11

Nowadays, negative pressure wound therapy is a widely used method in complex wound care 10 There are not many publications about NPWT in necrotizing fasciitis. Some authors suggest using NPWT only a few days after the primary operation. 16 Others reported good results with prompt use of negative pressure wound therapy ${ }^{3}$ In the presented case the prompt use of NPWT for extensive necrotizing fasciitis of the upper extremity was successful. The NPWT provided also a positive effect on the acute compartment syndrome of the

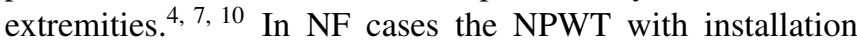


(NPWTi) seems to be more effective than conventional NPWT ${ }^{49}$ In the presented case the authors didn't use installation, because the first necrosectomy seemed to be complete. The NPWT-assisted skin traction can facilitate the primary closure of the skin. ${ }^{11}$ The used pressure is recommended between -100 and $-450 \mathrm{mmHg}$, but the most commonly used negative pressure is $-125 \mathrm{mmHg}$, 1, 34 6 9 In the present case the authors used $-125 \mathrm{mmHg}$ negative pressure with continuous suction.

Despite the complex surgical, antibiotic and intensive care the mortality rate in patients with necrotizing fasciitis is still high up to $75-100 \%$. 1.6. 11

\section{REFERENCES}

[1] J. S. Shin and H. J. Choi, "Application of a silicone sheet in negative-pressure wound therapy to treat an abdominal wall defect after necrotizing fasciitis," Archives of plastic surgery, vol. 44, no. 1, p. 76, 2017.

[2] P. Schipper and B. H. Tieu, "Acute chest wall infections: Surgical site infections, necrotizing soft tissue infections, and sternoclavicular joint infection," Thoracic surgery clinics, vol. 27, no. 2, pp. 73-86, 2017.

[3] S.-j. Chen, Y.-x. Chen, J.-r. Xiao, X.-z. Wei, S.-m. Chen, and W.-z. Jiang, "Negative pressure wound therapy in necrotizing fasciitis of the head and neck," Journal of Oral and Maxillofacial Surgery, vol. 77, no. 1, pp. 87-92, 2019.
[4] R. Felte, K. E. Gallagher, G. H. Tinkoff, and M. Cipolle, "A case review series of christiana care health system's experience with negative pressure wound therapy instillation," Cureus, vol. 8, no. 11, 2016.

[5] C.-H. Wong, L.-W. Khin, K.-S. Heng, K.-C. Tan, and C.-O. Low, "The lrinec (laboratory risk indicator for necrotizing fasciitis) score: a tool for distinguishing necrotizing fasciitis from other soft tissue infections," Critical care medicine, vol. 32, no. 7, pp. 1535-1541, 2004.

[6] P. S. Corona, F. Erimeiku, M. M. Reverté-Vinaixa, F. Soldado, C. Amat, and L. Carrera, "Necrotising fasciitis of the extremities: implementation of new management technologies," Injury, vol. 47, pp. S66-S71, 2016.

[7] J. G. Skedros, J. S. Smith, M. K. Henrie, E. D. Finlinson, and J. D. Trachtenberg, "Upper extremity compartment syndrome in a patient with acute gout attack but without trauma or other typical causes," Case Reports in Orthopedics, vol. 2018, 2018.

[8] A. E. Andersson, I. Egerod, V. E. Knudsen, and A.-M. Fagerdahl, "Signs, symptoms and diagnosis of necrotizing fasciitis experienced by survivors and family: a qualitative nordic multi-center study," $B M C$ infectious diseases, vol. 18, no. 1, p. 429, 2018.

[9] D. F. Blazek and E. Lockridge, "Instillation therapy for the treatment of necrotizing fasciitis: a case study," Cureus, vol. 8, no. 12, 2016

[10] J. Apelqvist, C. Willy, A.-M. Fagerdahl, M. Fraccalvieri, M. Malmsjö, A. Piaggesi, A. Probst, and P. Vowden, "Ewma document: Negative pressure wound therapy: Overview, challenges and perspectives," Journal of wound care, vol. 26, no. Sup3, pp. S1-S154, 2017.

[11] J. Y. Lee, H. Jung, H. Kwon, and S.-N. Jung, "Extended negative pressure wound therapy-assisted dermatotraction for the closure of large open fasciotomy wounds in necrotizing fasciitis patients," World Journal of Emergency Surgery, vol. 9, no. 1, p. 29, 2014. 